\title{
Spatial distribution of white matter degenerative lesions and cognitive dysfunction in relapsing-remitting multiple sclerosis patients
}

\author{
Natalia Nowaczyk ${ }^{1}$, Alicja Kalinowska-Łyszczarz ${ }^{2}$, Włodzimierz Paprzycki ${ }^{3}$, \\ Sławomir Michalak ${ }^{2}$, Radosław Kaźmierski ${ }^{4}$, Mikołaj A. Pawlak ${ }^{4}$ \\ ${ }^{1}$ Department of Health Psychology and Clinical Psychology, Institute of Psychology, Adam Mickiewicz University in Poznan, Poland \\ ${ }^{2}$ Division of Neurochemistry and Neuropathology, Department of Neurology, Poznan University of Medical Sciences (PUMS), \\ 49 Przybyszewskiego Street, Poznan, Poland \\ ${ }^{3}$ Department of Neuroradiology, Poznan University of Medical Sciences, Poland \\ ${ }^{4}$ Department of Neurology and Cerebrovascular Disorders, Poznan University of Medical Sciences, L. Bierkowski Hospital, Poznan,
} Poland

\begin{abstract}
Aim. The aim of this study was to assess degenerative lesion localisation in the course of relapsing-remitting multiple sclerosis (RRMS) and to identify the association between localisation and the frequency of T1-hypointense lesions (black holes) with cognitive dysfunction. We also searched for neuroradiological predictors of cognitive dysfunction in patients. The clinical rationale for the study was previous research, and our own findings suggest that lesion localisation plays an important role in cognitive performance and neurological disability of MS patients.

Material and methods. Forty-two patients were included in the study. All subjects underwent neuropsychological examination using Raven's Coloured Progressive Matrices, a naming task from the Brief Repeatable Battery of Neuropsychological Tests, and attention to detail tests. Magnetic resonance imaging (MRI) was acquired on 1.5 Tesla scanner and black holes were manually segmented on T1-weighted volumetric images using the FMRIB Software Library. Linear regression was applied to establish a relationship between black hole volume per lobe and cognitive parameters. Bonferroni correction of voxelwise analysis was used to correct for multiple comparisons.

Results. The following associations between black hole volume and cognition were identified: frontal lobes black hole volume was associated with phonemic verbal fluency $(t=-4.013, p<0.001)$, parietal black hole volume was associated with attention $(t=-3.776, p<0.001)$, and parietal and temporal black hole volumes were associated with nonverbal intelligence $(p<0.001)$. The volume of parietal black holes was the best predictor of cognitive dysfunction.

Conclusions. Our approach, including measurement of focal axonal loss based on T1-volumetric MRI sequence and brief neuropsychological assessment, might improve personalised diagnostic and therapeutic decisions in clinical practice.
\end{abstract}

Key words: relapsing-remitting multiple sclerosis (RRMS), T1-hypointense lesions, black holes, magnetic resonance imaging (MRI), cognitive dysfunction

(Neurol Neurochir Pol 2019; 53 (1): 18-25)

Address for correspondence: Natalia Nowaczyk, Department of Health Psychology and Clinical Psychology, Institute of Psychology, Adam Mickiewicz University in Poznan, Szamarzewskiego 89, 60-568 Poznan, Poland, e-mail: natalia.nowaczyk@amu.edu.pl 


\section{Introduction}

Multiple sclerosis (MS) is the most common autoimmune disease of the central nervous system (CNS) in young adults. It is defined as a chronic neuroinflammatory and neurodegenerative disease, which in its most typical course leads to relapses and remissions of various neurological symptoms, resulting from focal demyelinating lesions of the CNS [1]. Magnetic resonance imaging (MRI) is a key tool in MS diagnosis, monitoring, measuring treatment response and predicting disability. While disease activity is usually related to T2 or FLAIR hyperintense white matter lesions, or gadolinium enhancing T1 lesions, the neurodegenerative aspect of MS is represented by $\mathrm{T} 1$ hypointense lesions with high water content. These are called black holes [2]. Depending on the degree of neuronal damage, black holes can be classified as persistent, transitional or slowly evolving lesions [3-4]. Some of the focal degenerative changes may be transformed into persistent black holes [2, 5-6]. Early evolution of severe neurodegenerative lesions indicates more aggressive and rapidly disabling disease [7].

Currently, treatment guidelines do not address the occurrence of new black holes as an indication to escalate therapy, despite the fact that those lesions are irreversible and over time are followed by brain atrophy. Black holes represent complete demyelination with irreversible axonal damage on the focal level. As such, they are likely to contribute to disease progression, which has been shown in several studies [8-9]. Also, their clinical relevance may be reflected by their associations with other MRI parameters (lower supratentorial and infratentorial brain volume) [10], clinical data (disease duration, score of EDSS) [10-11] and cognitive impairment [12].

In the course of multiple sclerosis, $40-70 \%$ of patients develop cognitive dysfunction [13]. The most commonly observed deficits related to MS include executive dysfunction, low verbal fluency, and difficulties in visuospatial performance, short-term memory, abstract reasoning and attention [14-15]. These may be accompanied or related to depression or general fatigue. Several MRI parameters have been correlated with cognitive dysfunction in MS patients. While total white matter T2 lesion volume load is a modest correlate for MS- related cognitive impairment [16], measures of neurodegeneration, including brain atrophy, are stronger predictors of cognitive dysfunction [17-18]. These measures include black holes, but also other MRI parameters that correlate with cognitive dysfunction and clinical markers of disability, such as fractional anisotropy (FA) or mean diffusivity (MD) coefficient, derived from diffusion tensor imaging (DTI) methods [19]. Similar results have been obtained by using functional MRI or magnetisation transfer (MT) imaging [20]. These techniques, although potentially superior, require additional scanning time and advanced post-processing, which makes black holes assessment a more feasible yet reliable method.

The purpose of the present study was to assess the spatial distribution of MS-related white matter neurodegenerative lesions and its association with cognitive impairment, focusing on the following domains: nonverbal intelligence, attention, and phonemic verbal fluency. We hypothesised there is an association between localisation and the frequency of black holes and examined cognitive functions, and that black holes in the brain lobes are predictors of cognitive functioning.

\section{Clinical rationale for the study}

Early detection of neurodegenerative changes in multiple sclerosis should prompt a change in patient therapy. However, current treatment guidelines do not indicate any specific actions based on detection of new black holes. We evaluated the relationship between black holes in selected cortical regions of the brain and the severity of cognitive deficits.

The complete extent of brain damage cannot be estimated without a precise neurocognitive assessment. Neglecting the cognitive component of patient dysfunction might result in missing an important insight into disease progression, and the main reason behind the decline of quality of life in young individuals with MS.

\section{Materials and methods}

\section{Cohort characteristics}

Forty-two patients (25 women, 17 men) with relapsing-remitting multiple sclerosis fulfilling the $2010 \mathrm{McDonald}$ criteria [21] at the time of diagnosis were recruited for the study. The mean disease duration was 5.6 years (range $0-24$ ). Four patients were diagnosed at the time of inclusion into the study, while the longest time since diagnosis was 24 years in one patient. Age and EDSS measures are presented in Table 1. Thirty six percent of patients had obtained higher education. Significant comorbidities were found in three subjects: bronchial asthma $(\mathrm{n}=2)$ and ulcerative colitis $(\mathrm{n}=1)$. Beck Depression Inventory (BDI) scores ranged from 0 to 39 (median $=7$, interquartile range, $I Q R=1-12$ ). Exclusion criteria included: current relapse stage or relapse within the last eight weeks, immunomodulatory therapy in the last year, age older than 65 years, concomitant psychiatric disease, current use of neuroleptics or antiepileptic drugs, alcohol or drug abuse, diagnosis of another autoimmune or neoplastic pathology, relevant motor deficit in the dominant hand, upper limb ataxia or loss of visual acuity, and dementia. The neurological, MRI and neuropsychological examinations were all performed within a one month period. Patients treated with intravenous steroids within the previous three months, or immunomodulation at any point before baseline assessment, were not included in the study.

\section{Procedure}

All subjects underwent a neurological examination, psychometric assessment and MRI examination. Psychometric tests used for the assessment of cognition included:

- Raven's Coloured Progressive Matrices (RCPM; non-verbal intelligence) [22] 
- Attention to detail test (attention) [23]

- Spontaneous word list generation test, which is a modified version of phonemic naming task from Brief Repeatable Battery of Neuropsychological Tests (phonemic verbal fluency).

Structural MRI was performed on 1.5 Tesla MRI scanner (Siemens Avanto, Erlangen, Germany), with the use of Magnetisation Prepared Rapid Acquisition Gradient Echo (MPRAGE, $\mathrm{TR}=2400 \mathrm{~ms}, \mathrm{TE}=3.61 \mathrm{~ms}, \mathrm{IR}=1000 \mathrm{~ms}$, slice thickness $1.2 \mathrm{~mm}$, pixel band width $180 \mathrm{~Hz}$, FOV $240 \times 240 \mathrm{~mm}$, voxel size $1.25 \times 1.25 \times 1.20 \mathrm{~mm})$ and FLAIR space sequence $(\mathrm{TR}=$ $6000 \mathrm{~ms}, \mathrm{TE}=359 \mathrm{~ms}, \mathrm{IR}=2200 \mathrm{~ms}$, slice thickness $1.5 \mathrm{~mm}$, pixel band width $930 \mathrm{~Hz}$, FOV $227 \times 260 \mathrm{~mm}$, voxel size $1.01 \times 1.01 \times 1.50 \mathrm{~mm})$.

Structural analysis of T1-weighted sequences was performed with the use of FSL (FMRIB Software Library, https://fsl.fmrib. ox.ac.uk/fsl; version 5.0.6) [24] installed on Linux (Ubuntu 16.04 LTS). The analysis included: manually segmenting black holes by a reader blinded to clinical status, reproduction of manual segmentation after four weeks, and Bland-Altman assessment of intra-rater reproducibility. Probabilistic brain MNI152 atlas included in the FSL package was registered to each patient using flirt and black hole volume from each lobe was extracted using the fslstats tool.

Manual segmentation allowed the creation of masks of the black holes (dark area in T1-weighted sequences). Detailed detection of images was possible with the use of the fslview tool. Before the brain extraction procedure, the masks of degenerative changes in white matter were created by using the fslview and fslmerge (to concatenate the images) technique.

Secondly, brain and structure extraction were performed by the fslroi command and Brain Extraction Tool (BET) of FSL. Fslroi command allows extraction of a selected region of the brain based on the determination of the size values of individual axes. BET methods were needed to delete non-brain tissue from images of the whole head. We also estimated the inner and outer skull surfaces, and outer scalp surfaces, for good quality T1 input images. Two parameters were used: the force threshold $(\mathrm{f}=0.44$ ) and the gradient threshold $(g=0)$. The level of the force threshold was optimised. The duration of the brain extraction process was up to one minute.

A registration process was necessary to fit extracted scans and masks to the MN152 atlas of the FSL program. Before masks registration, the binarisation procedure (masks value $=1$, other brain structure $=0$, default value of threshold) was conducted using the fslmaths technique. In our linear registration, the parameters were set on 12 degrees of freedom (three rotations, three translations, three scales, and three warps), the cost function (such as mutual information), and three-linear interpolation. The result of this affine transformation process was a linear transformation matrix needed to receive standard images nearing to the atlas template. After the registration, the masks and scans were matched using the ApplyXFM option.

Brain lobes were selected based on the structural atlas MN152 of brain template images. Automated image registration algorithms were used to align brain MRI images with the target image [25]. By using the MN152 brain atlas (the volumetric coordinate system created by averaging MRI scans of 152 people) it was possible to analyse nine anatomical structures: frontal, parietal, occipital and temporal lobes, thalamus, insula, caudate, putamen and cerebellum. For the result of linear registration for each scan, see Figure 1.

\section{Statistical analysis}

Non-parametric mapping software (NPM) was used to analyse all scans with fitted plaques' masks in the MNI152 atlas. In cases of binarised masks, we used a Voxel Based Morphometry (VBM) technique in MRICRON ver. 2012 software. We calculated statistical maps for each cognitive dysfunction and black hole spatial distribution. Each variable was evaluated using a Shapiro-Wilk normality test. Normally distributed variables
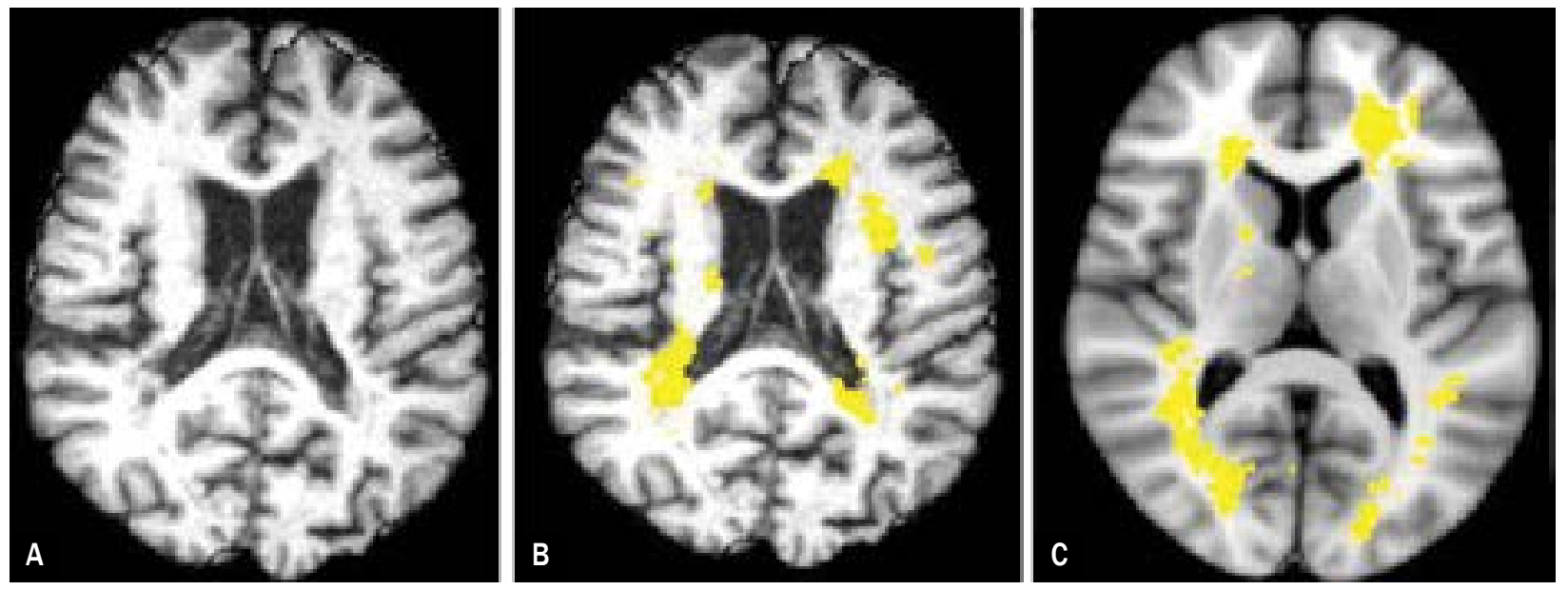

Figure 1. A linear registration of multiple sclerosis plaques and scans of axial brain section in magnetic resonance imaging (MRI): (A) extracted brain, (B) extracted brain after linear registration with prepared masks, (C) previous image fitted to the MNI152 atlas 
were summarised using mean and standard deviation. For non-normally distributed continuous variables, median and interquartile range (IQR) were calculated. Finally, linear regression analysis and stepwise regression analysis were conducted to identify associations between black hole volume per lobe and cognitive dysfunctions. BPF was calculated according to the formula: grey and white matter volume / total intracranial volume derived from FSL FAST. We applied Bonferroni correction for multiple comparisons. We also calculated Spearman's correlation coefficients for BPF and global black hole volume. Statistical analyses were performed using the RCRAN statistical environment (www.r-project.org) and the RStudio 1.1.453 graphical interface.

\section{Results}

\section{Descriptive statistics for variables in the study}

The descriptive statistics for all the measured variables are presented in Table 1.

\section{Spatial distribution of degenerative lesions}

Lesion distribution maps for nonverbal intelligence, phonemic verbal fluency and attention are represented in Figure 2.

\section{Lobar black hole lesion load}

Spatial heterogeneity of black hole location limits the clinical interpretation of the VBM results. Therefore, we calculated black hole lesion load for each lobe and conducted stepwise regression to identify the lobe where the lesion load was the best predictor of cognitive dysfunction.

Stepwise linear regression performed using black hole lesion load identified parietal lobe damage as the main predictor of cognitive dysfunction for each domain. Parietal lesion load predicted 2 to $29 \%$ of variance in the cognitive functioning. Detailed results are presented in Table 2. BPF did not correlate with total black hole lesion load ( $r h o=-0.161, p=0.348$ ), but there was a correlation with the temporal black hole load (rho $=-0.344, p=0.040)$. No significant differences $(p>0.05)$ of the volumetric parameters were found for gender or level of education in the studied MS cohort.

\section{Discussion}

We found that in RRMS cohort parietal lobe black hole lesion load was predicting cognitive dysfunction for each evaluated domain: attention, phonemic verbal fluency, and nonverbal intelligence. The role of the parietal lobe is focused on multimodal information integration, which is crucial for intelligence and attention control. Both these functions are frequently impaired in multiple sclerosis patients [26]. We observed the highest lesion load in parietal lobes, and the lowest in occipital lobes. The results of voxel-wise analysis identified lesions associated with dysfunction in other structures as well. Specifically, attention
Table 1. Descriptive statistics for clinical data of the examined group

\begin{tabular}{lcc} 
Variable & $\begin{array}{c}\text { Median(IQR) or } \\
{ }^{*} \text { Mean(SD) }\end{array}$ & Range \\
\hline Age & ${ }^{*} 39.55(11.3)$ & $18-63$ \\
EDSS & $1.00(0-2.0)$ & $0-6$ \\
Cognitive functions & & \\
Phonemic verbal fluency & ${ }^{*} 20.05(4.38)$ & $12-31$ \\
Nonverbal intelligence & $33(30-35)$ & $20-36$ \\
Attention & $7(5-8)$ & $1-8$ \\
Volumetric measurements & & \\
BPF & ${ }^{*} 0.77(0.03)$ & $0.70-0.85$ \\
Spatial distribution of black hole load per lobe [cm $]$ & \\
Frontal lobes & $0.62(0.18-2.39)$ & $0.00-15.23$ \\
Parietal lobes & $1.10(0.09-3.45)$ & $0.00-15.63$ \\
Temporal lobes & $0.06(0.00-0.63)$ & $0.00-17.21$ \\
Occipital lobes & $0.26(0.00-1.71)$ & $0.00-10.00$ \\
All MS plaques & $7.02(1.82-16.48)$ & $0.21-87.05$
\end{tabular}

deficit was associated with caudate lesions, and nonverbal intelligence and phonemic verbal fluency deficits were associated with frontal lobe lesions.

In previous studies, the incidence of black holes explained disharmonious cognitive and emotional processing in MS [27]. The location of degenerative white matter lesions can be associated with subsequent development of atrophy in distant brain regions [28-31].

Most of the published studies have focused on the relationship between black holes and neurological disability measured with the EDSS scale [32]. However, black holes are also associated with cognitive dysfunction [33]. This aspect has been underestimated and only a few studies have provided detailed analysis of this association [16]. In one study, T1 hypointense lesions were shown to correlate with Stroop test performance [34]. In another study, which analysed cognitive event-related potentials (ERPs), white matter degenerative lesion volume was associated with semantic and phonemic verbal fluency [14]. Both the Stroop test and the verbal fluency test require high performance in executive functions. This suggests that spatial distribution of degenerative white matter lesions could influence executive functions and other cognitive domains, including attention or auditory processing [35]. Similar results were found by Hojjat et al. [12]. According to their study, there were regional perfusion associations with cognitive dysfunction.

As for the choice of the psychometric tests, we used easily accessible tools that could be applied in everyday clinical practice. Following the literature, we decided to assess cognitive functions that had already been described as being affected in the course of MS [36], namely attention, which we measured with the use of an attention to detail test [23], and non-verbal intelligence (involving the visuo-spatial processes), which we assessed with Raven's Coloured Progressive Matrices [22]. To assess the 


\section{Nonverbal intelligence}

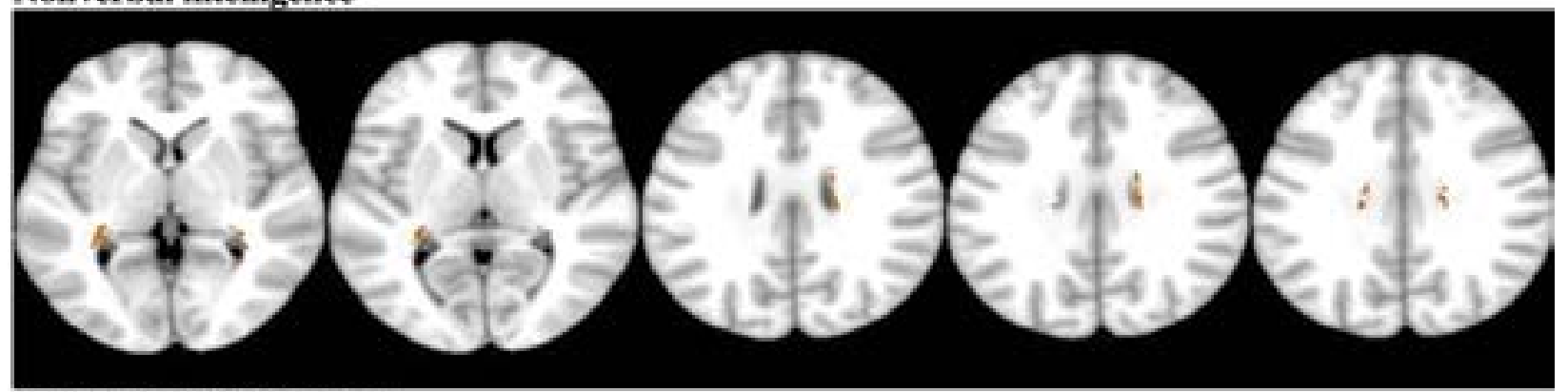

\section{Phonemic verbal fluency}
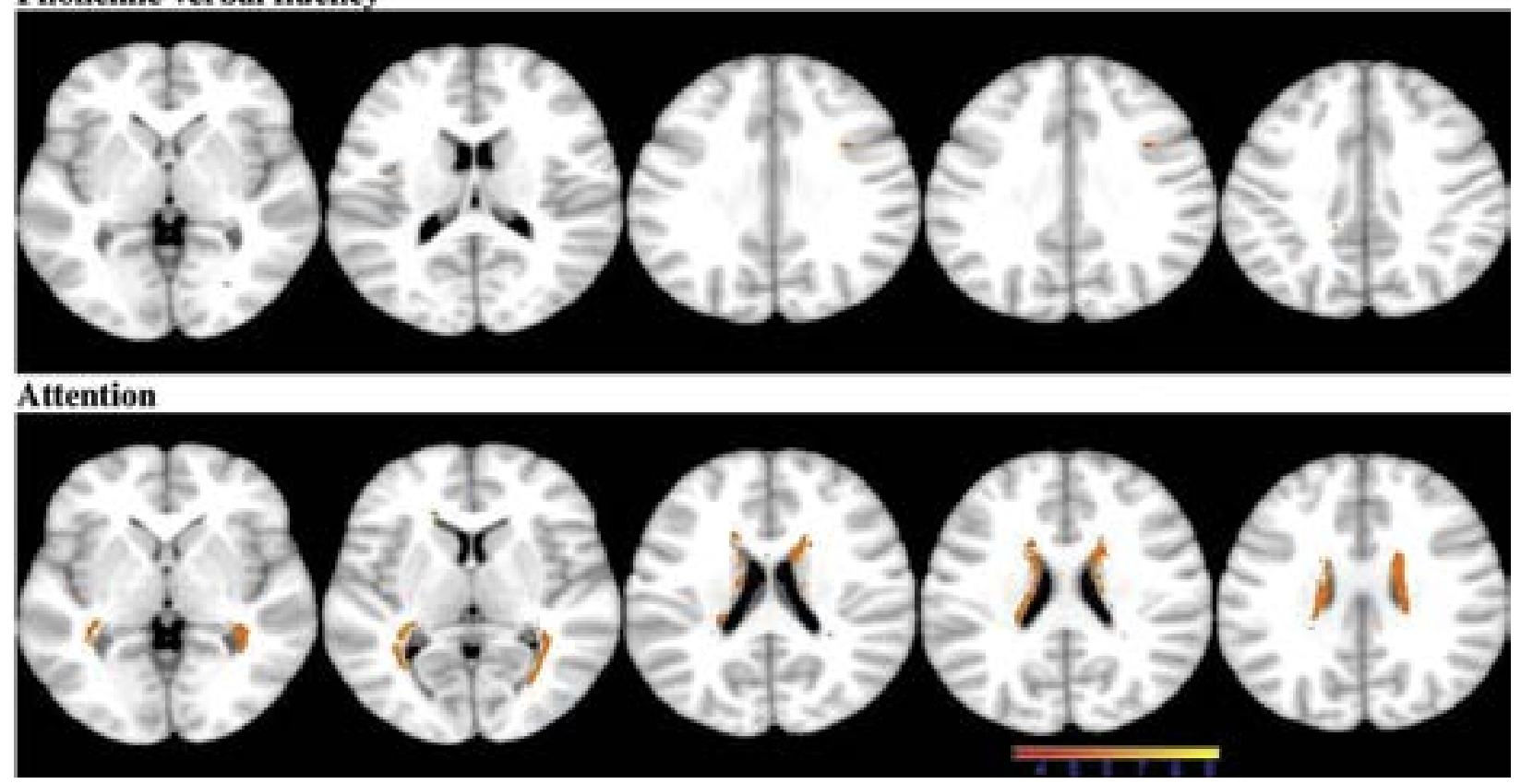

Figure 2. Statistical maps of black hole distribution for each cognitive function computed using Voxel Based Morphometry (VBM) analysis (Brunner-Munzel z statistics). Lesions are overlaid on the Montreal Neurological Institute (MNI) brain in axial slices. Colored voxels were $-\log$ of the $p$ value $(p<0.05)$. The equivalent of Bonferroni-corrected threshold was 5.112 and the equivalent of BM threshold was 3.1734 .

phonemic verbal fluency we used a modified version of the Word List Generation (WLG), which is a phonemic naming task in the original BRB-N [37]. WLG is a semantic verbal fluency test. In our study, we decided to measure one type of verbal fluency. Phonemic verbal fluency, which consists in generating words for a given letter in a minute, requires higher efficiency of executive functions than semantic fluency [38-40].

Individual lesions in multiple sclerosis always run the chance of damaging critical infrastructure and causing widespread dysfunction of structures distant to the lesion. This is often hard to prove because of low lesion burden per studied group. To solve that problem, grouping lesions based on atlas information might confer meaningful and easy-to-obtain information for every clinician. Modern image processing software is perfectly capable of producing a black hole lesion report per lobe, which in turn conveys the risk of cognitive dysfunction.
As for our methodological approach, the advantage of conducting linear registration using FMRIB's Linear Image Registration Tools (FLIRT) is that it may be fully automated, although it is significant to analyse the default values of the parameters. Also, according to Tam et al. [41] the assessment of the association between black holes volume and clinical parameters will be more precise if unpaired segmentation and paired registration methods are used. Literature data confirm that both fully-automated [42] and semi-automated [43] segmentation methods are justified. Manual lesion segmentation, such as we applied in the current study, is time consuming, and so in clinical practice its usefulness may be limited. Automated methods of black holes analysis are more likely to be used in everyday practice.

In the current study we chose to investigate white matter black holes. While it would be interesting to relate cognitive dysfunction also to cortical lesions in our cohort, this was not possible due to the sequence protocol and magnet field that we 
Table 2. Linear regression analysis of T1- weighted black holes in multiple sclerosis patients

\begin{tabular}{|c|c|c|c|c|c|}
\hline Volumes of black holes in brain structures & $R^{2}$ adjusted & $\mathbf{F}$ & $\boldsymbol{\beta}$ & $\mathbf{t}$ & $\mathbf{p}$ \\
\hline \multicolumn{6}{|l|}{ Phonemic verbal fluency } \\
\hline Frontal lobes & 0.269 & 16.108 & -0.536 & -4.013 & .000 \\
\hline Parietal lobes & 0.233 & 13.476 & -0.502 & -3.671 & .001 \\
\hline Temporal lobes & 0.132 & 7.221 & -0.391 & -2.687 & .010 \\
\hline Occipital lobes & 0.039 & 2.679 & -0.251 & -1.637 & .109 \\
\hline \multicolumn{6}{|l|}{ Attention } \\
\hline Frontal lobes & 0.100 & 5.542 & -0.349 & -2.354 & .024 \\
\hline Parietal lobes & 0.244 & 14.257 & -0.513 & -3.776 & .001 \\
\hline Temporal lobes & 0.107 & 5.895 & -0.358 & -2.428 & .020 \\
\hline Occipital lobes & 0.088 & 4.940 & -0.332 & -2.223 & .032 \\
\hline \multicolumn{6}{|l|}{ Nonverbal intelligence } \\
\hline Frontal lobes & 0.195 & 10.954 & -0.464 & -3.310 & .002 \\
\hline Parietal lobes & 0.290 & 17.732 & -0.554 & -4.211 & .000 \\
\hline Temporal lobes & 0.283 & 17.157 & -0.548 & -4.142 & .000 \\
\hline Occipital lobes & 0.098 & 5.456 & -0.346 & -2.336 & .025 \\
\hline
\end{tabular}

used. Cortical pathology is difficult to visualise in a conventional MRI protocol. Although the value of grey matter pathology has been increasing, as shown by the inclusion of cortical localisation in the latest version of MS criteria [44], it is still largely underestimated due to technical difficulties in their visualisation. Neither T2*-weighted gradient echo nor Double Inversion Recovery (DIR) sequences are routinely available. Also, high-field magnets, which are also more sensitive to cortical pathology, are not at a routine disposal. Nevertheless, cortical lesions have in fact been shown to be associated with cognition and disability in MS patients [45].

Our study is limited by its small, relatively heterogeneous sample size and relatively long and differential disease duration. The sample size was limited by the exclusion criteria, including immunomodulation therapy, which is currently widely available for RRMS and thus the sample size is limited. However, we wanted to avoid the possible influence of immunomodulatory drugs on brain volumetry results. Also, we focused solely on degenerative white matter lesions without taking into account FLAIR lesions and atrophy accumulated over time. This reductionist approach stemmed from the context of this work, which was primarily a masters' thesis of the first author. Another limitation is the choice of basic psychometric tests to assess cognitive dysfunction. However, this could also be a potential advantage, as they can be used without charge or time consumption in an everyday clinical practice setting.

\section{Clinical implications and future directions}

The pathophysiological substrate of cognitive dysfunction in MS is not fully understood. Several MRI markers have been suggested as predictors of cognitive impairment. Over recent years, attention has shifted to more and more sophisticated parameters derived from advanced image analysis. In this paper, we are revisiting the aspect of traditional and relatively easily identified $\mathrm{T} 1$ hypointense lesions detected on volumetric T1-weighted images as predictors of poor cognitive performance. We suggest that modern image processing software should be used to obtain a black hole lesion mapping report. Such a report could aid in assessing the individual risk of cognitive dysfunction in MS patients, and in selecting the patients for detailed neuropsychological assessment. Also, linking cognitive dysfunction to degenerative white matter lesions could be an important predictor of MS patients' quality of life. It may aid in selecting patients in need of neuropsychological rehabilitation [46].

Studies on a larger sample size and using standardised batteries of neuropsychological tests are needed to enable the use of black hole spatial distribution analysis in everyday clinical practice.

Conflict of interest: NN and WP: No conflict of interest declared. AKŁ received personal compensation for speaking services and/or travel cost compensation and/or research grants from Biogen, Bayer, Merck Serono, Novartis, Teva Pharmaceuticals, CSL Behring, Shire, Sanofi-Genzyme, Roche. SM received personal compensation for speaking services and/or travel cost compensation and/or research grants from Bayer, TEVA, Novartis, Lundbeck, Biogen Idec and EUROIMMUN. RK received speaking honoraria and/or consultancy fees and/or travel expenses from Biogen Idec, Novartis, and SanofiGenzyme. MAP received speaking honoraria and/or travel engagements and/or research grants from Novartis, Roche, Biogen, Merck, Teva Pharmaceuticals. 
Acknowledgement and financial support: Alicja Kalinowska-Lyszczarz was supported by National Science Centre grants 2012/05/D/NZ6/00989 and 2012/07/B/NZ6/03529. Mikolaj A. Pawlak was supported by National Science Centre grant 2011/01/D/NZ4/05801. We would like to thank our patients who participated in the study.

Ethics: This study was approved by the Internal Review Board at the Poznan University of Medical Sciences. Written informed consent was obtained from all the study participants.

\section{References}

1. Rovira A, Montalban X. MR brain iron mapping in MS: a potential neurodegenerative marker or just another technique? Neurology. 2011; 77(18): 1660-1661, doi: 10.1212/WNL.0b013e318236f120, indexed in Pubmed: 21975201.

2. Sahraian MA, Radue EW, Haller S, et al. Black holes in multiple sclerosis: definition, evolution, and clinical correlations. Acta Neurol Scand. 2010; 122(1): 1-8, doi: 10.1111/j.1600-0404.2009.01221.x, indexed in Pubmed: 20003089.

3. Zhang $\mathrm{Y}$, Traboulsee A, Zhao Y, et al. Texture analysis differentiates persistent and transient T1 black holes at acute onset in multiple sclerosis: a preliminary study. Mult Scler. 2011; 17(5): 532-540, doi: 10.1177/1352458510395981, indexed in Pubmed: 21270058.

4. Elliott C, Wolinsky JS, Hauser SL. Detection and characterisation of slowly evolving lesions in multiple sclerosis using conventional brain MRI. Multiple Sclerosis Journal. 2017; 23: 52-53.

5. Papadopoulou A, von Felten S, Traud S, et al. Evolution of MS lesions to black holes under DNA vaccine treatment. J Neurol. 2012; 259(7): 1375-1382, doi: 10.1007/s00415-011-6361-x, indexed in Pubmed: 22222856.

6. van Walderveen MA, Kamphorst W, Scheltens P, et al. Histopathologic correlate of hypointense lesions on T1-weighted spin-echo MRI in multiple sclerosis. Neurology. 1998; 50(5): 1282-1288, indexed in Pubmed: 9595975.

7. Mitjana R, Tintoré M, Rocca MA, et al. Diagnostic value of brain chronic black holes on T1-weighted MR images in clinically isolated syndromes. Mult Scler. 2014; 20(11): 1471-1477, doi: 10.1177/1352458514526083, indexed in Pubmed: 24576831.

8. Truyen L, Waesberghe Jv, Walderveen Mv, et al. Accumulation of hypointense lesions ("black holes") on T1 spin-echo MRI correlates with disease progression in multiple sclerosis. Neurology. 1996; 47(6): 1469-1476, doi: 10.1212/wnl.47.6.1469.

9. Paolillo A, Coles AJ, Molyneux PD, et al. Quantitative MRI in patients with secondary progressive MS treated with monoclonal antibody Campath 1H. Neurology. 1999; 53(4): 751-757, indexed in Pubmed: 10489036.

10. Paolillo A, Pozzilli C, Gasperini C, et al. Brain atrophy in relapsing-remitting multiple sclerosis: relationship with 'black holes', disease duration and clinical disability. J Neurol Sci. 2000; 174(2): 85-91, indexed in Pubmed: 10727693.

11. Rossi F, Giorgio A, Battaglini M, et al. Relevance of brain lesion location to cognition in relapsing multiple sclerosis. PLoS One. 2012; 7(11): e44826, doi: 10.1371/journal.pone.0044826, indexed in Pubmed: 23144775.

12. Hojjat SP, Cantrell CG, Carroll TJ, et al. Perfusion reduction in the absence of structural differences in cognitively impaired versus unim- paired RRMS patients. Mult Scler. 2016; 22(13): 1685-1694, doi: 10.1177/1352458516628656, indexed in Pubmed: 26846987.

13. Rogers JM, Panegyres PK. Cognitive impairment in multiple sclerosis: evidence-based analysis and recommendations. J Clin Neurosci. 2007; 14(10): 919-927, doi: 10.1016/j.jocn.2007.02.006, indexed in Pubmed: 17659875.

14. Kimiskidis VK, Papaliagkas V, Sotirakoglou K, et al. Cognitive event-related potentials in multiple sclerosis: Correlation with MRI and neuropsychological findings. Mult Scler Relat Disord. 2016; 10: 192-197, doi: 10.1016/j.msard.2016.10.006, indexed in Pubmed: 27919489.

15. Rao SM, Leo GJ, Bernardin L, et al. Cognitive dysfunction in multiple sclerosis. I. Frequency, patterns, and prediction. Neurology. 1991; 41(5): 685-691, indexed in Pubmed: 2027484.

16. Rossi F, Giorgio A, Battaglini M, et al. Relevance of brain lesion location to cognition in relapsing multiple sclerosis. PLoS One. 2012; 7(11): e44826, doi: 10.1371/journal.pone.0044826, indexed in Pubmed: 23144775.

17. Zivadinov R, Jakimovski D, Gandhi S, et al. Clinical relevance of brain atrophy assessment in multiple sclerosis. Implications for its use in a clinical routine. Expert Rev Neurother. 2016; 16(7): 777-793, doi: 10.1080/14737175.2016.1181543, indexed in Pubmed: 27105209.

18. Kalinowska-Łyszczarz A, Pawlak MA, Pietrzak A, et al. Subcortical gray matter atrophy is associated with cognitive deficit in multiple sclerosis but not in systemic lupus erythematosus patients. Lupus. 2018; 27(4): 610-620, doi: 10.1177/0961203317735186, indexed in Pubmed: 28992796.

19. Tovar-Moll F, Evangelou IE, Chiu AW, et al. Diffuse and focal corticospinal tract disease and its impact on patient disability in multiple sclerosis. J Neuroimaging. 2015; 25(2): 200-206, doi: 10.1111/ jon.12171, indexed in Pubmed: 25318661.

20. van Munster CEP, Uitdehaag BMJ. Outcome Measures in Clinical Trials for Multiple Sclerosis. CNS Drugs. 2017; 31(3): 217-236, doi: 10.1007/s40263-017-0412-5, indexed in Pubmed: 28185158.

21. Polman $\mathrm{CH}$, Reingold SC, Banwell B, et al. Diagnostic criteria for multiple sclerosis: 2010 revisions to the McDonald criteria. Ann Neurol. 2011; 69(2): 292-302, doi: 10.1002/ana.22366, indexed in Pubmed: 21387374.

22. Carter P. The complete book of intelligence tests. John Wiley and Sons Ltd, The Atrium, Southern Gate, Chichester, West Sussex, England 2005.

23. Spreen 0 , Strauss EA. A compendium of neuropsychological tests. 2nd ed. Oxford University Press, New York, Oxford 1998.

24. Smith SM, Jenkinson M, Woolrich MW, et al. Advances in functional and structural MR image analysis and implementation as FSL. Neuroimage. 2004; 23 Suppl 1: S208-S219, doi: 10.1016/j.neuroimage.2004.07.051, indexed in Pubmed: 15501092.

25. Mandal PK, Mahajan R, Dinov ID. Structural brain atlases: design, rationale, and applications in normal and pathological cohorts. J Alzheimers Dis. 2012; 31 Suppl 3: S169-S188, doi: 10.3233/JAD2012-120412, indexed in Pubmed: 22647262.

26. Chiaravalloti ND, Stojanovic-Radic J, DeLuca J. The role of speed versus working memory in predicting learning new information in multiple sclerosis. J Clin Exp Neuropsychol. 2013; 35(2): 180-191, doi: 10.1080/13803395.2012.760537, indexed in Pubmed: 23350959.

27. Thaler C, Faizy T, Sedlacik J, et al. T1- Thresholds in Black Holes Increase Clinical-Radiological Correlation in Multiple Sclerosis Patients. PLoS One. 2015; 10(12): e0144693, doi: 10.1371/journal. pone.0144693, indexed in Pubmed: 26659852. 
28. Rocca MA, Comi G, Filippi M. The Role of T1-Weighted Derived Measures of Neurodegeneration for Assessing Disability Progression in Multiple Sclerosis. Front Neurol. 2017; 8: 433, doi: 10.3389/ fneur.2017.00433, indexed in Pubmed: 28928705.

29. Wartenburger I, Kühn E, Sassenberg U, et al. On the relationship between fluid intelligence, gesture production, and brain structure. Intelligence. 2010; 38(1): 193-201, doi: 10.1016/j.intell.2009.11.001.

30. Koziol JA, Wagner S, Sobel DF, et al. Asymmetries in the spatial distributions of enhancing lesions and black holes in relapsing-remitting MS. J Clin Neurosci. 2005; 12(8): 895-901, doi: 10.1016/j. jocn.2004.11.010, indexed in Pubmed: 16249086.

31. Batista S, Zivadinov R, Hoogs M, et al. Basal ganglia, thalamus and neocortical atrophy predicting slowed cognitive processing in multiple sclerosis. J Neurol. 2012; 259(1): 139-146, doi: 10.1007/s00415011-6147-1, indexed in Pubmed: 21720932.

32. Correale J, Peirano I, Romano L. Benign multiple sclerosis: a new definition of this entity is needed. Mult Scler. 2012; 18(2): 210-218, doi: 10.1177/1352458511419702, indexed in Pubmed: 21865415.

33. Thaler C, Faizy TD, Sedlacik J, et al. T1 Recovery Is Predominantly Found in Black Holes and Is Associated with Clinical Improvement in Patients with Multiple Sclerosis. AJNR Am J Neuroradiol. 2017; 38(2): 264-269, doi: 10.3174/ajnr.A5004, indexed in Pubmed: 28059711.

34. Ruggieri S, Fanelli F, Castelli L, et al. Lesion symptom map of cognitive-postural interference in multiple sclerosis. Mult Scler. 2018; 24(5): 653-662, doi: 10.1177/1352458517701313, indexed in Pubmed: 28337941.

35. Adusumilli G, Trinkaus K, Sun P, et al. Intensity ratio to improve black hole assessment in multiple sclerosis. Mult Scler Relat Disord. 2018; 19: 140-147, doi: 10.1016/j.msard.2017.11.020, indexed in Pubmed: 29223871.

36. Chiaravalloti ND, DeLuca J. Cognitive impairment in multiple sclerosis. The Lancet Neurology. 2008; 7(12): 1139-1151, doi: 10.1016/ S1474-4422(08)70259-X.

37. Huijbregts SCJ, Kalkers NF, de Sonneville LMJ, et al. Differences in cognitive impairment of relapsing remitting, secondary, and primary progressive MS. Neurology. 2004; 63(2): 335-339, indexed in Pubmed: 15277630.
38. Henry JD, Crawford JR. A meta-analytic review of verbal fluency performance following focal cortical lesions. Neuropsychology. 2004; 18(2): 284-295, doi: 10.1037/0894-4105.18.2.284, indexed in Pubmed: 15099151.

39. Piskunowicz M, Bieliński M, Zgliński A, et al. Testy fluencji słownej- zastosowanie w diagnostyce neuropsychologicznej. Psychiatria Polska. 2013; 47(3): 475-485.

40. Troyer AK, Moscovitch M, Winocur G, et al. Clustering and switching on verbal fluency: the effects of focal frontal- and temporal-lobe lesions. Neuropsychologia. 1998; 36(6): 499-504, indexed in Pubmed: 9705059.

41. Tam RC, Traboulsee A, Riddehough A, et al. Improving the clinical correlation of multiple sclerosis black hole volume change by paired-scan analysis. Neuroimage Clin. 2012; 1(1): 29-36, doi: 10.1016/j. nicl.2012.08.004, indexed in Pubmed: 24179734.

42. Tam RC, Traboulsee A, Riddehough A, et al. The impact of intensity variations in T1-hypointense lesions on clinical correlations in multiple sclerosis. Mult Scler. 2011; 17(8): 949-957, doi: 10.1177/1352458511402113, indexed in Pubmed: 21502309.

43. Thaler C, Faizy TD, Sedlacik J, et al. The use of multiparametric quantitative magnetic resonance imaging for evaluating visually assigned lesion groups in patients with multiple sclerosis. J Neurol. 2018; 265(1): 127-133, doi: 10.1007/s00415-017-8683-9, indexed in Pubmed: 29159467.

44. Thompson AJ, Banwell BL, Barkhof F, et al. Diagnosis of multiple sclerosis: 2017 revisions of the McDonald criteria. Lancet Neurol. 2018; 17(2): 162-173, doi: 10.1016/S1474-4422(17)30470-2, indexed in Pubmed: 29275977.

45. Harrison D, Roy S, Oh J, et al. Association of Cortical Lesion Burden on 7-T Magnetic Resonance Imaging With Cognition and Disability in Multiple Sclerosis. JAMA Neurology. 2015; 72(9): 1004-1012, doi: 10.1001/jamaneurol.2015.1241.

46. Bakirtzis C, loannidis P, Messinis L, et al. The Rationale for Monitoring Cognitive Function in Multiple Sclerosis: Practical Issues for Clinicians. Open Neurol J. 2018; 12: 31-40, doi: 10.2174/1874205X01812010031, indexed in Pubmed: 30008964 . 